\title{
Interactive comment on "Drought resistance increases from the individual to the ecosystem level in highly diverse neotropical rain forest: a meta-analysis of leaf, tree and ecosystem responses to drought" by Thomas Janssen et al.
}

\section{Anonymous Referee \#2}

Received and published: 28 January 2020

In this study, Janssen et al perform a meta-analysis to look to drought impacts on carbon and water exchange across scales ranging from leaf, to plant, to ecosystem in neotropical rainforests. In particular, the authors contrast physiological responses from seasonal water stress versus 'episodic' drought events. In doing this, the authors also look at wood density as a proxy for plant physiological responses. Its clear that the authors put in a significant amount of work through compiling 138 studies across 229 sites and this study will clearly contribute to the physiological literature on forest drought responses. I do have some major revision suggestions before publication which are 
summarized here and, in some cases, elaborated in the line specific comments below.

1) I think that the manuscript would benefit from reframing of the dry season 'drought' as a routine period of decreased water availability. When I think of droughts, I think of a prolonged period of abnormally low rainfall. Given that dry seasons occur every year, I don't see them meeting this definition. This reframing would provide a nice platform to discuss physiological responses to routine (seasonal) stress, such as phenology, versus episodic stress and can help get at important physiological mechanisms. This would involve some substantial reworking of the text, but I think it would really help the story line. 2) Some of the methods are confusing and I think elaborating a bit more, providing and providing a table might help. See line specific comments below 3) The Figure legends need to be more descriptive of all the features in the figures 4) Overall the manuscript does an impressive job discussing a range of processes, but the reader might be more attentive if it were a little shorter. Where possible, I would suggest the authors minimize extraneous discussion. In particular, I think talking about isohydricity requires significant motivation for a general audience (which is not provided), so I would cut this text.

Line-specific comments: L21-22 There is nothing to back up this statement on LSMs. I suggest the authors remove it

L35 Khanna et al 2017 Regional dry-season climate changes due to three decades of Amazonian deforestation

L50 after going through the MS, I am confused about where the multi-year drought is presented in the authors analysis

L53-54 This is exactly why I would argue that 'seasonal drought' is a misnomer

121 The concept of isohydricity/anisohydricity will likely not be familiar to a broad audience at Biogeosciences. I would encourage the authors to eliminate the jargon and focus on the mechanisms of interest (see Martinez-Vilalta 2016 "Water potential reg-

Printer-friendly version

Discussion paper 
ulation, stomatal behaviour and hydraulic transport under drought: deconstructing the iso/anisohydric concept") or else devote more space to describing isohydric behavior

L131-132 But the authors actually try and demonstrate that wood density is usable as a proxy first, so isn't this more of a hypothesis?

L145 where does the multiyear drought aspect come in that the authors mentioned in the intro?

Intro overall: The introduction is on the longer side (as is the manuscript) and the three separate sections do not have a smooth transition. I would try to combine 1.2 and 1.3 and streamline the text. Given that sections 1.2-1.3 are more about the mechanisms, I would put thes sections first and then say that different types of physiological stress also impact $\mathrm{C}$ and $\mathrm{W}$ fluxes including seasonal, routine decreases in water availability versus droughts

\section{L152 ERA5 citation?}

L153-155 It would be good for the authors to say how many studies were associated with each of these diagnostics and how many studies had multiple observations, maybe a table would be useful?

L157-159 I am confused about how observations were calculated/recorded across scales? Could the authors elaborate? For example, were ecosystem-level measurements independent from leaf level, or were different levels calculated using observations from different scales by the authors? Both more details and a table detailing number of measurements for each diagnostic and number of studies that cover multiple diagnostics and scales would help

L161-162, this doesn't need to be included

L166-167 This is a huge amount of work, I commend the authors

Discussion paper

L170 it would be good to say the spatial resolution and include a citation for on ERA5 
(30km?)

L171 monthly average midday VPD?

L187 how was this error estimated?

L219 Figures should be renumbered so Fig 2 comes after Fig 1

L208-209 It would be good to include a possible caveat about uncertainty associates with ERA5 soil moisture (which I presume is modeled)

L212-213 why the $65 \%$ and $10 \%$ quantiles? Did the authors test the sensitivity of their results to this assumption?

L220 Could a study be both a dry season and episodic drought? I am a little confused about the partitioning. Also, where does multi-year drought come in?

L225 did the authors check to see if it was necessary to log the response?

Where did the ENSO data come from?

L254 how can predawn wp be positive 0.22?! Please check for a typo

L281 Could the authors include Reco in some of the figures, they refer a change in Reco several times in the text but no visuals are provided

L363 typo include 'us'

\section{L370 denoted iWUE previously}

L401 it is also true that there are more observations post 2000. The authors should discuss how this might impact their results

L419 it would be nice to put these numbers in a physiological context

L451-458 I think there is a really nice opportunity to contrast tree physiological strategies when exposed to routine stress (the dry season) versus drought that the current narrative doesn't allow for when both are classified as drought 
L461 is it the short timescale, or that fact that the plants are used to this type of stress and use phenology to deal with it?

L464 Or maybe title: how do we scale from the leaf to the ecosystem? This is a universal problem in ecology that researchers across many subdisciplines will sympathize with

\section{L502 see previous suggestion about isohydricity}

L536 This isn't a great comparison. The authors of this manuscript analyze site-specific data whereas the spatial scale of the Konings study is $0(100 \mathrm{~km})$. For me, this paragraph does not contribute much to the study and in general I think the isohydricity framework is not useful here (and otherwise)

L575 LSMs are brought up in only in the abstract and conclusion. It doesn't add to the discussion and I would remove this

L582 How should they be used to benchmark LSMs? If the authors insist on including this, please the need to spell out the methodology rather than throwing it in as a concluding sentence

Other relevant citation: Detto 2018 "Resource acquisition and reproductive strategies of tropical forest in response to the El Niño-Southern Oscillation"

Figures: Fig. 1 panel a make lat/lon bigger b) its really hard for me to wrap my head around what the authors mean by this metric, can they elaborate? d) What do the dots mean? Please describe this in the legend and also detail what dark and light gray correspond to legend $(e)$ it would be good to remind the reader what the positive/negative ENSO index means L1131 "terrestrial" isn't capitalized

Fig. 2 can the authors denote the sample size above each category in the figure? For example, does $\mathrm{n}=3$ for Episodic drought soil-leaf hydraulic conductance in panel $b$ ? Make sure to describe the figure fully (detail quantile boxes, median line, error bars, and outliers) in the legend

Printer-friendly version

Discussion paper
Interactive

comment 
Fig. 3-4 I would combine these two figures into 12 -column 3-row figure. I generally really like this format and found it very effective in the Ainsworth review. Great job describing all aspects of the figure in the legend

Fig 5-6 I would combine these figures into a 2-c 3-r figure The point size is the inverse of the sample standard error of the effect size in the study. $\rightarrow$ so studies with a smaller SE have a larger dot? Moderator= independent variable?

Fig 5 legend, make sure to walk the reader though each panel

Interactive comment on Biogeosciences Discuss., https://doi.org/10.5194/bg-2019-497, 2020. 